\title{
Spatiotemporal Chaos Synchronization Based on the Combination Control of Local Function
}

\author{
Jingyun Jang, Gang Li, Jia Li, Juan Bao, Jia Hou \\ College of Physics and Electronic Technology \\ Liaoning Normal University, \\ Dalian 116029, China
}

\begin{abstract}
A method of the spatiotemporal chaos synchronization in globally coupled map lattice (CML) is proposed. The combination controller is designed and the range of the coupling constant of local function is obtained based on Lyapunov stability theory. The global synchronization of two globally CMLs is realized. Numerical simulation shows the effectiveness of the results.
\end{abstract}

Keywords-Spatiotemporal chaos synchronization, Local function, Combination control, Lyapunov function

\section{INTRODUCTION}

The coupled map lattice (CML) has been widely used on the investigation of spatiotemporal chaotic behavior because of its advantages of discrete-time, discrete-space and continuous-state. Since the CML model has important application in secure communication and automatic control, etc., it has become the hotspot in the chaotic control and synchronization. And many synchronization methods have been proposed, such as linear coupling method, nonlinear coupling method, adaptive control method, variable feedback method and so on [1-9]. Generally, the linear coupling method is a simple method and easy to be put in practice. The nonlinear coupling method is proposed for the synchronization of more complex spatiotemporal dynamical systems. An important advantage of this method is that the coupled signal is more flexible to design when the synchronization is realized. Therefore, theoretical and applied investigation on it is necessary and significant.

In this paper, a method of the spatiotemporal chaos synchronization in globally coupled map lattice (CML) is proposed. The combination controller is designed and the range of the coupling constant of local function is obtained based on Lyapunov stability theory. The global synchronization of two globally CMLs is realized. Numerical simulation shows the effectiveness of the results.

\section{SYNCHRONIZATION OF THE GLOBALLY CML}

\section{SYSTEMS}

The globally CML as a producer of spatiotemporal chaos is described as follows:

$$
x_{n+1}(i)=(1-\varepsilon) f\left(x_{n}(i)\right)+\frac{\varepsilon}{L-1} \sum_{j=1}^{L} f\left(x_{n}(j)\right)
$$

where $x_{n}(i)$ is the state variable, $n$ is the discrete time, $i$ is the lattice site index $(i=1,2 \ldots . L), L$ is the system size, and $\varepsilon$ is the coupling strength factor of discrete lattice point. The logistic map is taken as the local function:

$$
f\left(x_{n}(i)\right)=a x_{n}(i)\left(1-x_{n}(i)\right)
$$

where $a$ is the system parameter.

The phase map of the globally CML at each lattice site is showed in Figure.1, when the size of the system is 100, the initial condition is a random value. The system parameters are given as $a=4.0, \varepsilon=0.1$, and the periodic boundary condition is given as $x_{n}(0)=x_{n}(L)=0$.

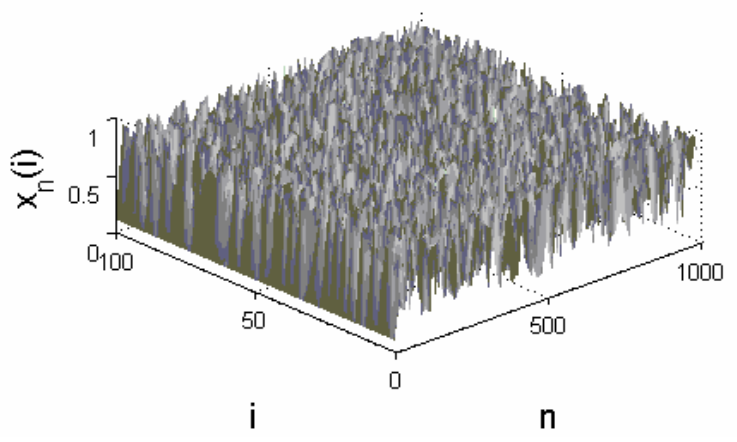

Figure 1. Phase map of the globally CML

The system (1) is taken as the target system which can be classified into a nolinear part and the other part:

$$
x_{n+1}(i)=f\left(x_{n}(i)\right)+H\left(x_{n}(i)\right)
$$

where

$$
H\left(x_{n}(i)\right)=(-\varepsilon) f\left(x_{n}(i)\right)+\frac{\varepsilon}{L-1} \sum_{j=1 j \neq i}^{L} f\left(x_{n}(j)\right)
$$

Another globally CML system with the same structure is used as the response system:

$$
\begin{aligned}
y_{n+1}(i) & =f\left(y_{n}(i)\right)+H\left(y_{i}(i)\right)+u_{n} \\
& =f\left(y_{n}(i)\right)+H\left(x_{i}(i)\right)+\frac{k}{a}\left(f\left(x_{n}(i)\right)-f\left(y_{n}(i)\right)\right)
\end{aligned}
$$

where $u_{n}$ is the conbination controller,

$$
u_{n}=H\left(x_{i}(i)\right)-H\left(y_{i}(i)\right)+\frac{k}{a}\left(f\left(x_{n}(i)\right)-f\left(y_{n}(i)\right)\right)
$$


and $k$ is the coupling constant of local function.

The synchronization error of the two CML systems is defined as:

$$
e_{n}(i)=x_{n}(i)-y_{n}(i)
$$

And the Lyapunov function can be taken as follows :

$$
V=\left|e_{n}(i)\right|
$$

obviously,

$$
\begin{aligned}
& \Delta V=\left|e_{n+1}(i)\right|-\left|e_{n}(i)\right|=\left|x_{n+1}(i)-y_{n+1}(i)\right|-\left|e_{n}(i)\right| \\
& =\left|\left(1-\frac{k}{a}\right)\left(f\left(x_{n}(i)\right)-f\left(y_{n}(i)\right)\right)\right|-\left|e_{n}(i)\right| \\
& =\left(\left|(a-k)\left(1-\left(x_{n}(i)+y_{n}(i)\right)\right)\right|-1\right)\left|e_{n}(i)\right| \\
& \text { where } \quad 0 \leq x_{n}(i) \leq 1 \quad, \quad 0 \leq y_{n}(i) \leq 1 \quad \text {, so } \\
& \left|1-\left(x_{n}(i)+y_{n}(i)\right)\right| \leq 1 \text {. } \\
& \text { If }-1 \leq(a-k) \leq 1 \\
& \text { i.e. } \\
& \left|(a-k)\left(1-\left(x_{n}(i)+y_{n}(i)\right)\right)\right| \leq 1
\end{aligned}
$$

It's easy to see from the equation above

$$
\Delta V \leq 0
$$

According to Lyapunov stability theory, when $n \rightarrow \infty$, the error state variable will approach to zero gradually. The global synchronization of the two CMLs is realized.

\section{SIMULATIONS}

The parameters of the system are given as $\varepsilon=0.1, a=4.0$ and $L=100$. The periodic boundary conditions are given as $x_{n}(0)=x_{n}(L)=0$ and $y_{n}(0)=y_{n}(L)=0$. The initial conditions are two random values. We start exerting the combination control on the response system at step $n=2000$. The spatiotemporal evolution of the synchronization error can be shown in Figures.2-3 by adjusting the coupling constant at $k=3.1$ and $k=4.9$.

From Figures.2-3 we can see that the orbits of the two systems have nothing to do with each other before exerting the controller. But after the step $n=2000$, the synchronization error $e_{n}(i)$ approaches to zero quickly with spatiotemporal evolution, which means the synchronization of the two spatiotemporal chaotic systems is realized. And the synchronization effectiveness is good.

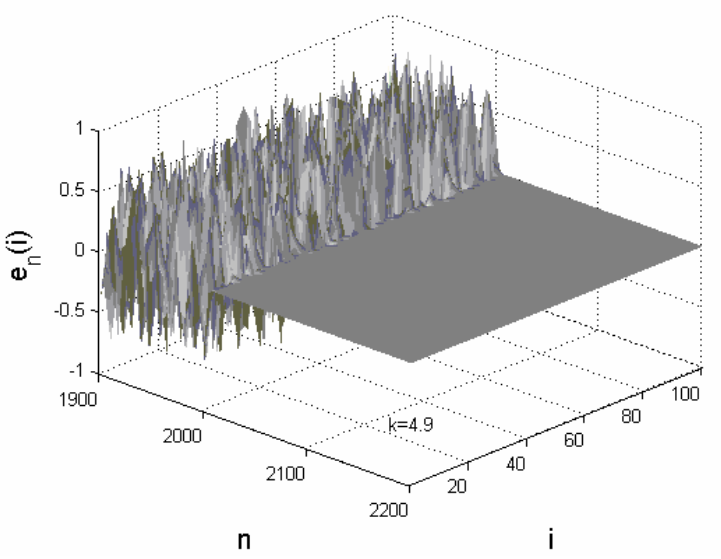

Figure 2. Spatiotemporal evolution of the error $(k=3.1)$

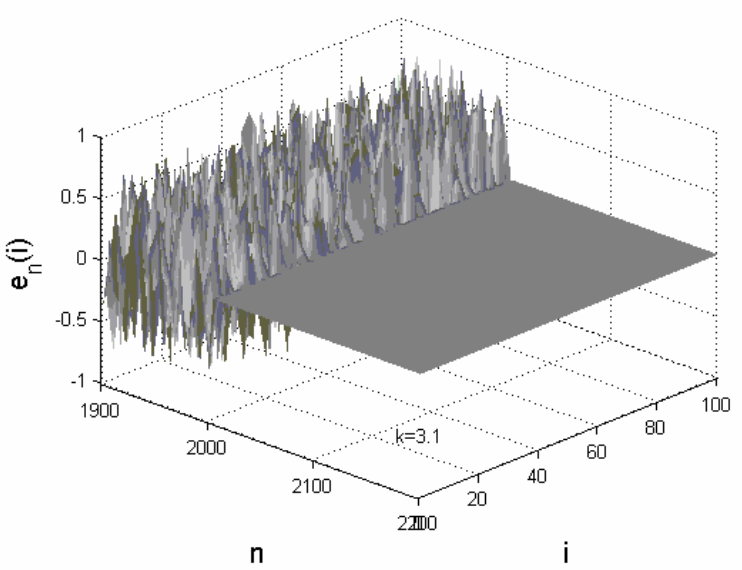

Figure 3. Spatiotemporal evolution of the error $(k=4.9)$

\section{CONCLUSIONS}

In this paper, the spatiotemporal chaos synchronization in globally CML is realized by using the combination control method. The combination controller is designed and the range of the coupling constant is obtained based on Lyapunov stability theory. Numerical simulation shows that the synchronization error approaches to zero quickly and the result is effective.

\section{REFERENCES}

[1] JIANG Pin-Qun; WANG Bing-Hong; XIA Qing-Hua; BU ShouLiang. Control spatio-temporal chaos in coupled map lattice by state feedback. Acta Physica Sinica [J], 2004.53, PP3280-3286.

[2] WANG Jin-lan; CHEN Guang-zhi. Spatiotemporal chaos synchronization by active-occasional coupling. Acta Physica Sinica [J], 1999.9, PP1605-1610.

[3] LIU Jian-bo; YE Chun-fei; ZHANG Shu-jing; SONG Wen-tao. Antiphase synchronization in coupled map lattices. Physics Letters A [J], 2000.274, PP27-29.

[4] HE Guo-yi; HE Guo-wei. Synchronous chaos in the coupled system of two logistic maps. Chaos, Solitons \& Fractals [J], 2004.23, PP909913. 
[5] ]HUANG Li-lian; WANG Mao; FENG Ru-peng. Synchronization of generalized Henon map via backstepping design. Chaos, Solitons \& Fractals[J], 2005.23, PP617-620.

[6] DEILAMIA M Z; CHERATI Z R; MOTLAGH M R J. Control of spatio-temporal on-off intermittency in random driving diffusively coupled map lattices. Chaos, Solitons \& Fractals[J], 2007.41, PP113122.

[7] RAHMANI Z; MOTLAGH M R J. Adaptive control of spatiotemporal chaos in coupled map lattices. Chaos, Solitons \& Fractals [J], 2009.41, PP1697-1707.

[8] LÜ Ling, XIA Xiao-lan. Anti-synchronization of nonlinear-coupled spatiotemporal chaotic system. Acta Physica Sinica [J], 2009, PP814818.

[9] LÜ Ling; LI Gang; CHAI Yuan. The synchronization of spatiotemporal chaos of unilateral coupled map lattice. Acta Physica Sinica[J], 2009.2, PP7517-7522. 